International Journal of Medical Sciences

ISSN 1449-1907 www.medsci.org 2006 3(4):130-134

Case Report

(c)2006 Ivyspring International Publisher. All rights reserved

\title{
Malignant phyllodes tumor with heterologous liposarcomatous differentiation and tubular adenoma-like epithelial component
}

\author{
L. Uriev ${ }^{1}$, I. Maslovsky ${ }^{2}$, P. Vainshtein ${ }^{3}$, B. Yoffe ${ }^{3}$, D. Ben-Dor ${ }^{1}$ \\ 1. Institute of Pathology, Barzilai Medical Center, Ashkelon, Israel \\ 2. Department of Internal Medicine, Barzilai Medical Center, Ashkelon, Israel \\ 3. Department of General and Vascular Surgery, Barzilai Medical Center, Ashkelon, Israel
}

Correspondence to: Dr. Igor Maslovsky, phone 972-8-8558048; fax 972-8-8558057; email: igorgn@012.net.il or igorgn@barzi.health.gov.il

Received: 2006.03.20; Accepted: 2006.07.21; Published: 2006.08.15

Phyllodes tumor of the breast is a biphasic fibroepithelial neoplasm. A 30-year-old woman presented with a 1year history of a palpable, asymptomatic right breast mass without axillary lymphadenopathy and family history of breast carcinoma. Malignant phyllodes tumor was diagnosed. The authors present not previously described histological appearance of this tumor where an epithelial component was identical to that of a tubular adenoma of the breast, with the review of the literature. This is in addition to very rare liposarcomatous stromal differentiation in the malignant phyllodes tumor.

Key words: phyllodes tumor, liposarcoma, tubular adenoma

\section{Introduction}

Phyllodes tumors are rare entity in the breast. They make up less than $1 \%$ of all breast tumors [1]. The majority of these lesions behave in a completely benign fashion in that they do not have metastatic potential, leaving local recurrence as the only real concern. Local recurrence per se is not an indicator of malignancy because it has been described in benign, borderline, and malignant phyllodes tumors.

Phyllodes tumors are characterized by a combination of hypercellular stroma and cleft-like or cystic spaces lined by epithelium, into which the stroma classically project in a leaf-like fashion. There are wide variation in histological appearances between different tumors, ranging from those which resemble fibroadenomas, apart from increased stromal cellularity and mitotic activity, to those showing a diffuse overgrowth of highly pleomorphic stromal cells resembling a soft tissue sarcoma, with a spectrum of appearances intermediate between these extremes [2].

\section{Report of a case}

A 30-year-old woman presented with a 1-year history of a palpable, asymptomatic right breast mass. There was no family history of breast carcinoma. No axillary lymphadenopathy was identified. Mammography and ultrasound revealed well circumscribed mass in the lower outer quadrant of the right breast. The excisional biopsy was performed. Malignant phyllodes tumor has been diagnosed. The tumor was completely excised. Clinical follow-up for 12 months has not revealed any evidence of focal disease recurrence, metastasis or any mass in the contralateral breast.

\section{Pathologic findings}

Two specimens measured $6 \times 2.7 \times 1.5 \mathrm{~cm}$ and 2.8 $\times 1.7 \times 1.5 \mathrm{~cm}$, total weight of $30 \mathrm{~g}$, with smooth outer surface have been received. Cut sections showed homogenous, tan-yellow tissue of an elastic consistency.

Microscopic examination of both specimens exhibited biphasic proliferation with pushing border, in which the stromal components displayed remarkable overgrowth (Fig.1). The stromal component was predominantly represented by adipose tissue with numerous hyperchromatic stromal cells and lipoblasts showing marked pleomorphism and atypia (Fig.2). Mitotic count in these areas was 2-3 per 10 high power fields. Highly atypical stromal cells were also intermingled with epithelial component. Only few foci with spindle cell stroma and leaf-like projections were seen (Fig.3). Malignant phyllodes tumor with heterologous liposarcomatous differentiation was diagnosed.

The epithelial component was of particular interest. It demonstrated a proliferation of moderately dilated compact, rounded regular glands, partially with eosinophilic proteinaceous material, without atypia or mitotic activity, typically seen in a tubular adenoma of the breast (Fig.4,5). These glands contained a prominent myoepithelial cell layer what was confirmed by immunohistochemical stains.

\section{Discussion and review}

Phyllodes tumors of the breast are fibroepithelial neoplasms that have the potential for recurrence and metastases. Grading into benign, borderline, and malignant categories is based on a constellation of 
histological characteristics that include the degree of stromal hypercellularity, stromal cytologic atypia and mitotic activity, stromal overgrowth, and circumscribed vs. invasive margins [2]. Although histological features have been helpful to some extent in predicting biologic behavior, specific parameters that can define the likelihood for recurrence are not universally accepted. Various investigators have found cellular pleomorphism, stromal overgrowth, tumor necrosis and heterologous stromal elements, or a combination of histological features to be prognostically useful. On the other hand, several authors have concluded that adequacy of surgical margins is of paramount importance and that histological factors have an inconsistent influence on biologic behavior [3].

Sarcomatous stromal elements, including angiosarcoma, chondrosarcoma, leiomyosarcoma, osteosarcoma, and rhabdomyosarcoma, are rarely encountered in malignant phyllodes tumors [4]. Liposarcomas may also develop as stromal components of phyllodes tumors. Liposarcomatous differentiation in phyllodes tumors may consist of well differentiated, myxoid, round cell, and pleomorphic liposarcomatous elements [5]. The finding of a malignant heterologous element places the tumor into a malignant category [3].

On gross examination, a typical phyllodes tumor is notable for leaf-like fronds projecting into cystic spaces. Many phyllodes tumors, however, are simply solid and vaguely lobulated. Well developed fronds consist of epithelial-lined stromal projections that protrude into dilated glands or cystic spaces. The glands are usually widely spaced, dilated, and irregular with prominent side branches. The epithelium is often hyperplastic, and atypical columnar cell hyperplasia is a common finding. Rare tumors show lobular and ductal carcinoma in-situ. Apocrine and squamous metaplasia is occasionally seen [6-8].

To the best of our knowledge, we are reporting the first case in which an epithelial component of phyllodes tumor is identical to that of a tubular adenoma of the breast. And this is in addition to very rare liposarcomatous stromal differentiation in malignant phyllodes tumor. This tumor does not represent a collision tumor, i.e. tubular adenoma being immediately adjacent to the phyllodes tumor, because macroscopically cut section did not show separate masses, and microscopically lipoblasts widely infiltrated among epithelial glands. This tumor further expends the phenotypic features of phyllodes tumor.

\section{Acknowledgments}

This particular case was kindly examined by C.D.M. Fletcher, M.D., Professor of Pathology, Harvard Medical School and I. Bleiweiss, M.D., Professor of Pathology, Mount Sinai School of Medicine.

\section{Conflict of interests}

The authors have declared that no conflict of interest exists.

\section{References}

1. Carter BA, Page DL. Phyllodes tumor of the breast: local recurrence versus metastatic capacity. Hum Pathol. 2004; 35: 1051-1052.

2. Moffat CJC, Pinder SE, Dixon AR, Elston CW, Blarney RW, Ellis IO. Phyllodes tumors of the breast: a clinicopathological review of thirty-two cases. Histopathology. 1995; 27: 205-218.

3. Tan PH, Jayabaskar T, Chuan KL et al. Phyllodes tumors of the breast: the role of pathologic parameters. Am J Clin Pathol. 2005; 123: 529-540.

4. Rosen PP. Fibroepithelial neoplasms. In: Weinberg RW, Donnellan K, Palumbo R, eds. Rosen's Breast Pathology, 2nd ed. Philadelphia: Lippincott Williams \& Wilkins, 2001: 176-200.

5. Isotalo PA, George RL, Walker R, Sengupta SK. Malignant phyllodes tumor with liposarcomatous differentiation. Arch Pathol Lab Med. 2005; 129: 421-422.

6. Lerwill MF. Biphasic lesions of the breast. Semin Diagn Pathol. 2004; 21: 48-56.

7. Knudsen PJT, Ostergaard J. Cystosarcoma phylloides with lobular and ductal carcinoma in situ. Arch Pathol Lab Med 1987; 111: 873-875.

8. Nishimura R, Hasebe $T$, Imoto $S$, Mukai K. Malignant phylloides tumour with a non-invasive ductal carcinoma component. Virchows Arch. 1998; 432: 89-93. 
Int. J. Med. Sci. 2006, 3

Figures

Fig.1. Panoramic view of the tumor showing tubular and malignant adipose components (H\&E, x 40)

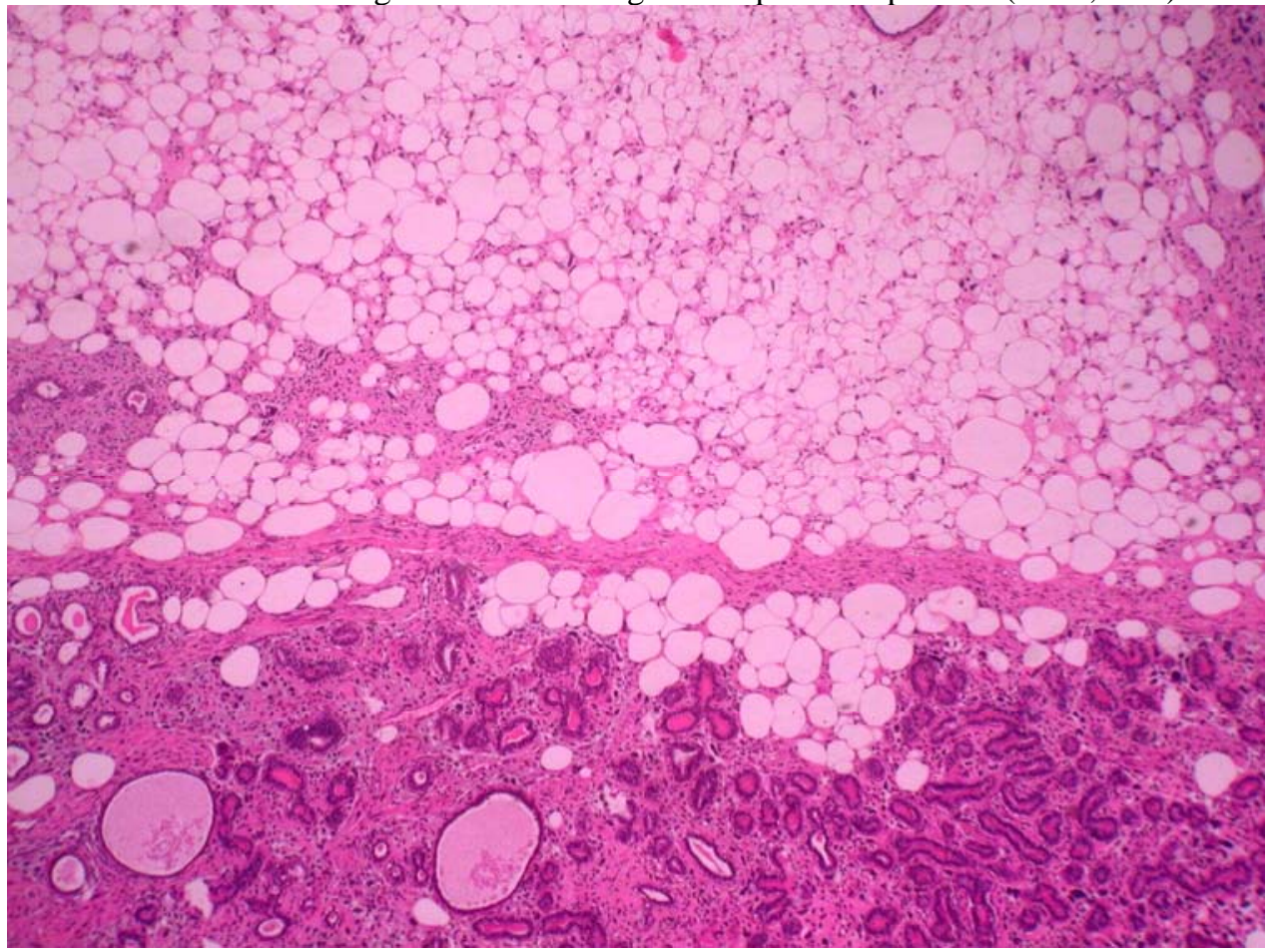

Fig.2. Stromal component with liposarcomatous differentiation (H\&E, x 100)

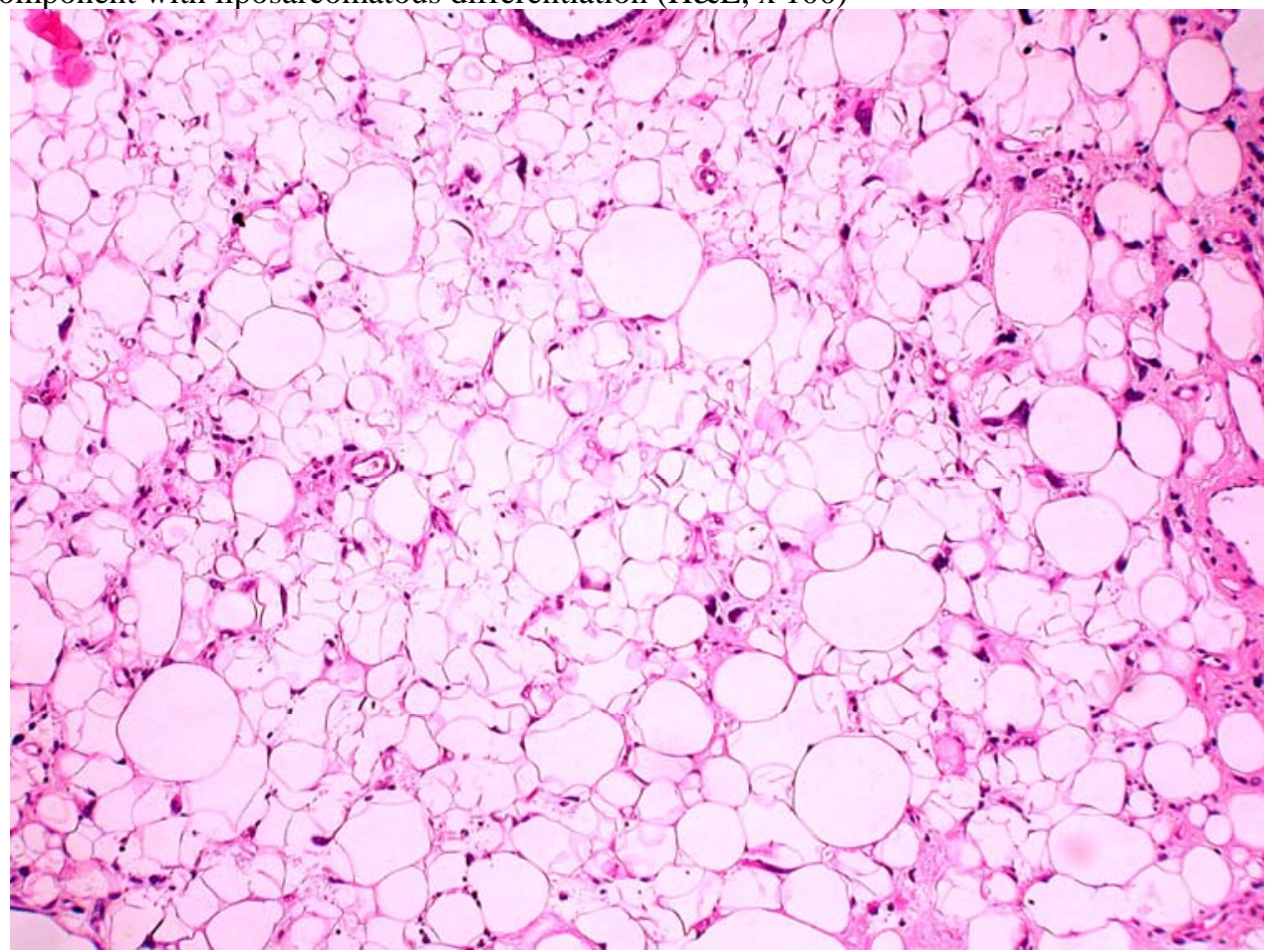


Int. J. Med. Sci. 2006, 3

Fig.3. Leaf-like projections characteristic of phyllodes tumor (H\&E, x 40)

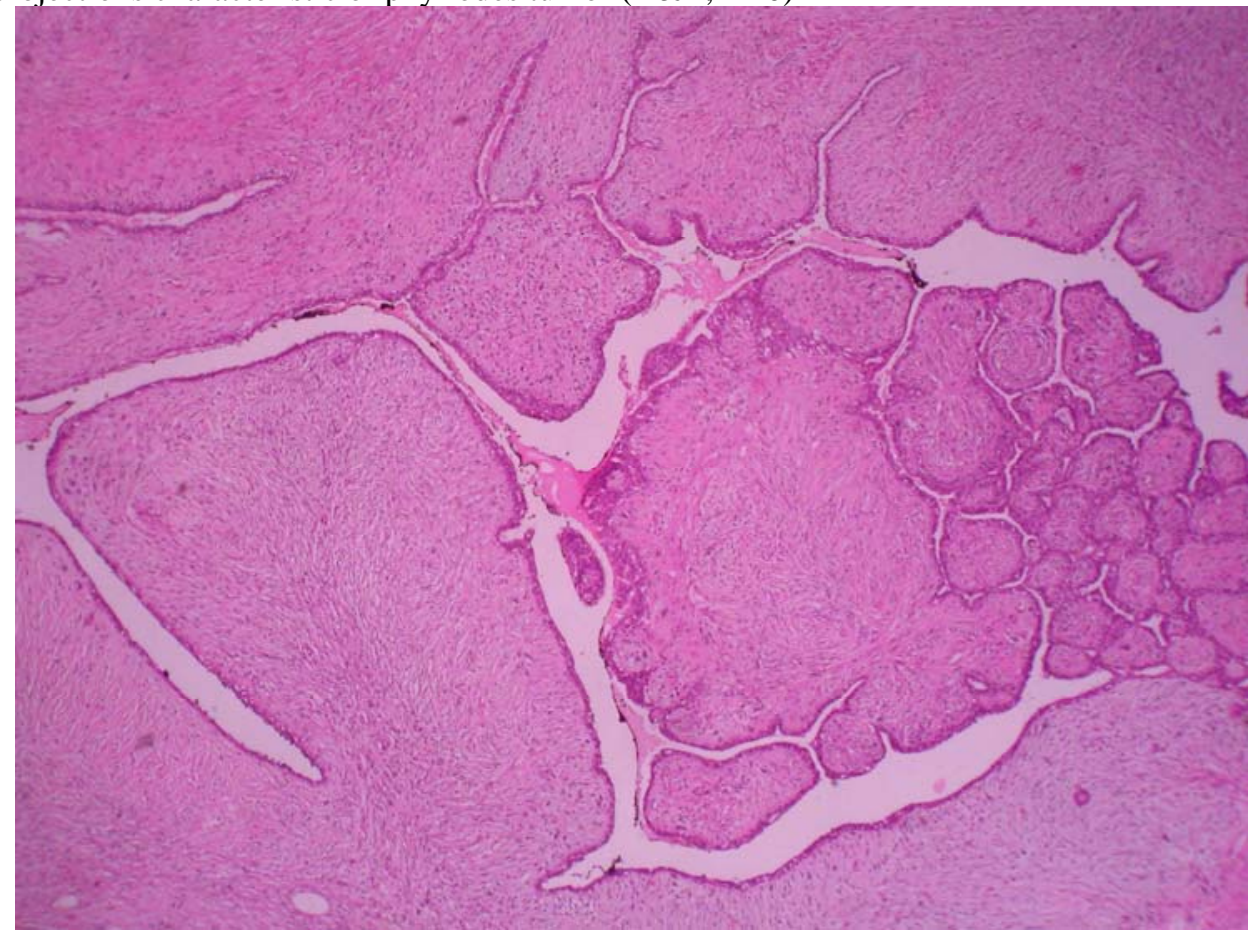

Fig.4. Tubular component with juxtaposed regular mammary lobules (H\&E, x 40)

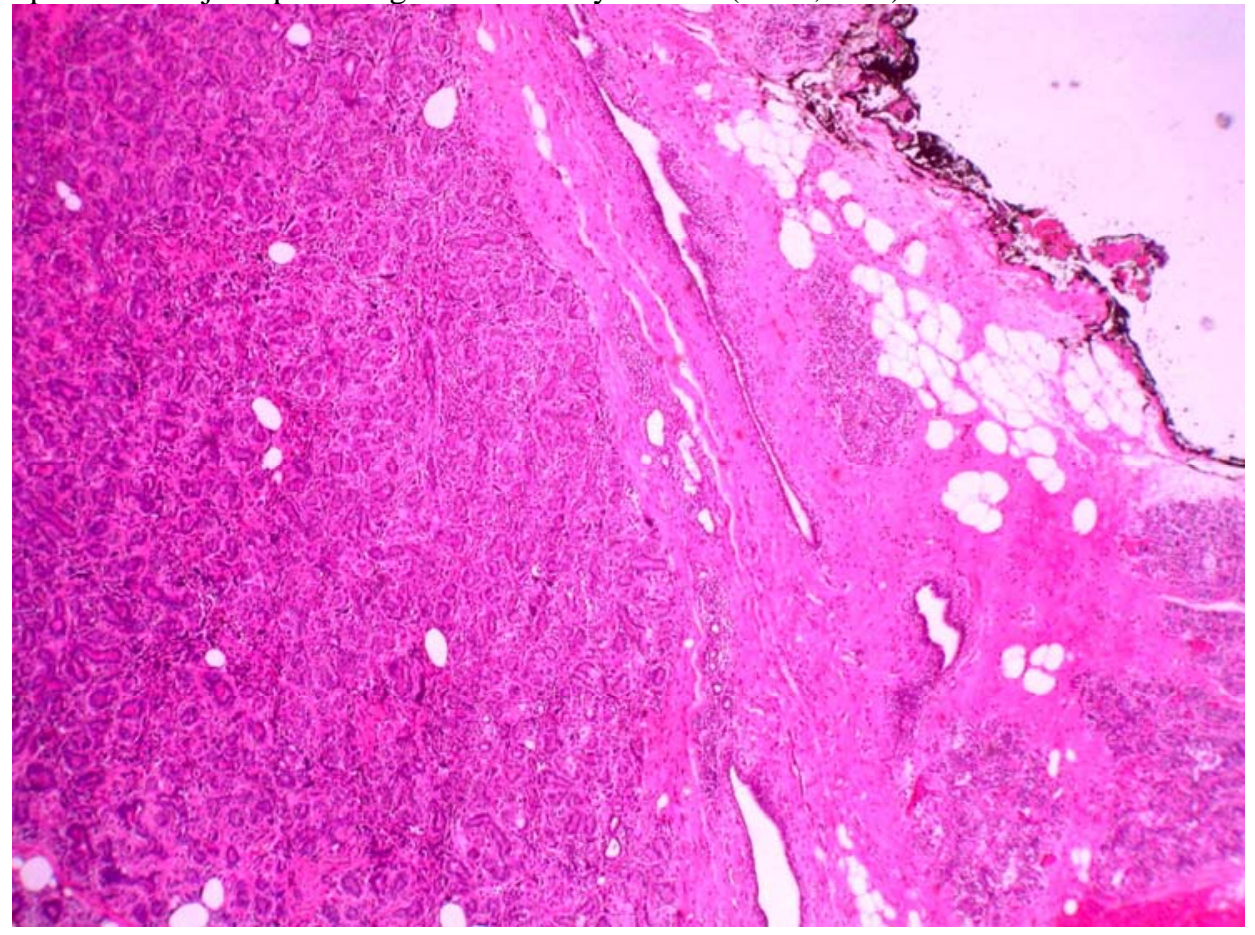


Int. J. Med. Sci. 2006, 3

Fig.5. Close-up of the tubular component (H\&E, x 200)

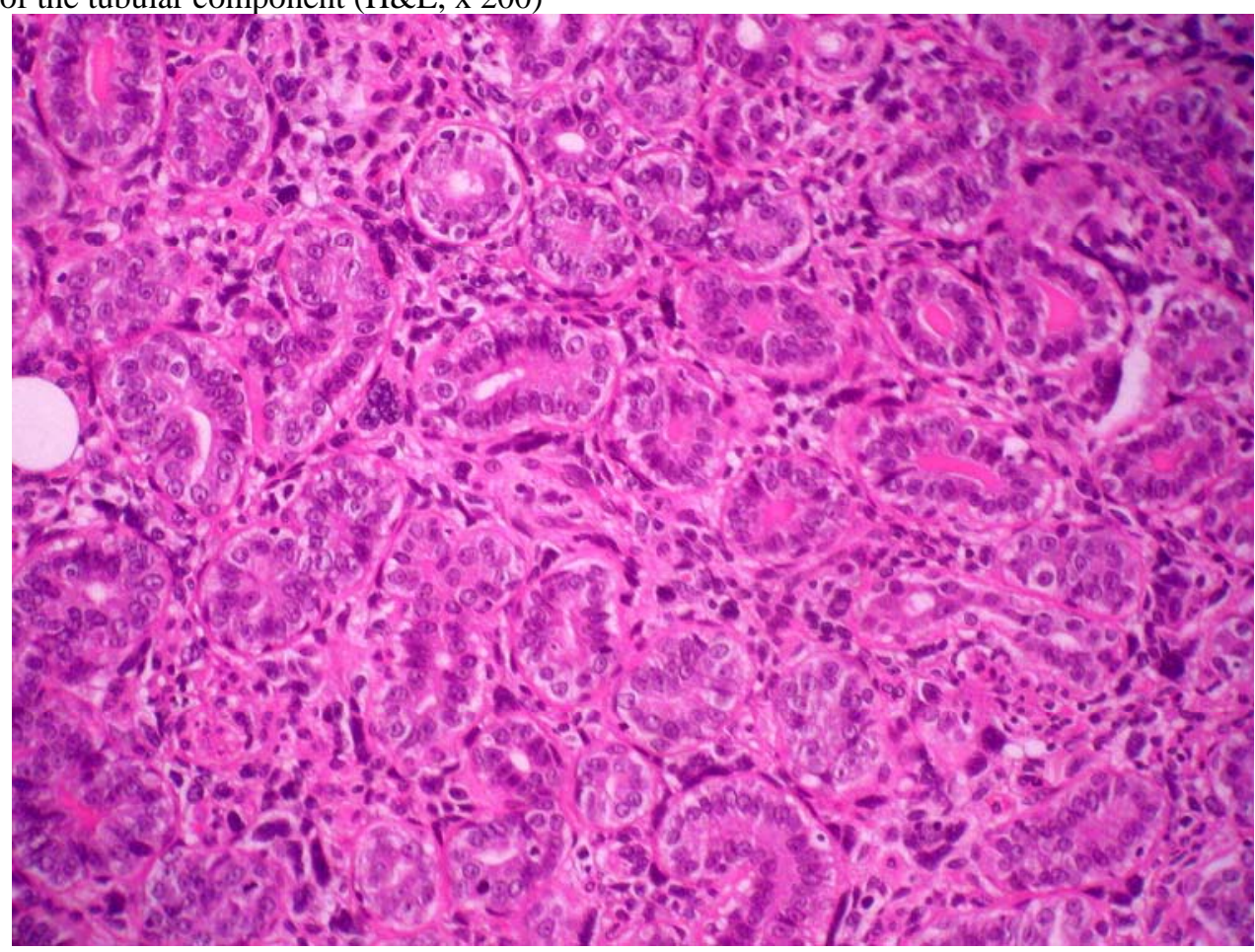

